\title{
Clínica de cães com cardiomiopatia dilatada idiopática, tratados ou não com carvedilol
}

\author{
Clinic of dogs with dilated cardiomyopathy (DCM) treated or not by carvedilol
}

\author{
Moacir Leomil Neto ${ }^{\mathrm{I}}$ Júlio César Carvalho Balieiro $^{\mathrm{II}}$ Elaine Cristina Soares Pereira ${ }^{\mathrm{III}}$ \\ Guilherme Gonçalves Pereira ${ }^{\text {II }}$ Valéria Marinho de Oliveira" ${ }^{\text {II }}$ Maria Helena Matiko Akao Larsson"
}

RESUMO

$O$ tratamento convencionalmente preconizado para cães acometidos pela CMD consiste na prescrição de vasodilatadores, agentes inotrópicos positivos (digitálico), diuréticos, dieta hipossódica e, quando necessário, antiarrítmicos. $O$ carvedilol é um $\beta$-bloqueador de $3^{a}$ geração, não seletivo, que bloqueia igualmente e competitivamente os receptores $\left(\beta_{1}, \beta_{2}, e \alpha_{1}\right)$. Produz uma evidente vasodilatação periférica, exerce efeitos anti-oxidantes, removendo radicais livres de oxigênio e prevenindo a peroxidação lipídica nas membranas cardíacas, prevenindo a perda de miócitos e a ocorrência de arritmias e reduzindo a taxa de mortalidade em pacientes humanos. $O$ objetivo do presente estudo foi avaliar clínica, eletrocardiográfica, radiográfica e ecocardiograficamente a evolução de cães com cardiomiopatia dilatada (CMD) tratatos com terapia convencional associada ao carvedilol. Para tal foram avaliados 49 cães com CMD divididos em: grupo NT, tratado com terapia convencional, $e$ grupo $T$, tratado com terapia convencional associada ao carvedilol. Os animais foram submetidos à avaliação clínica e a exames complementares durante o período de um ano. Os resultados demonstraram que a terapia com carvedilol apresentou boa tolerabilidade na dose de $0,3 \mathrm{mg} \mathrm{kg}^{-1} 12$ 12 horas, aumentou a sobrevida dos cães em 30,9\%, não alterou as pressões sistólica e diastólica, reduziu a frequência cardíaca após três semanas de terapia, melhorou significantemente as frações de encurtamento e ejeção após seis meses de tratamento, não promoveu alterações radiográficas e da distância E-septo, diminuiu o índice de letalidade da doença, fato demonstrado pela melhora no escore clínico e na classe funcional dos animais, obtida após três semanas de terapia com carvedilol.

Palavras-chave: cães, avaliação clínica, beta-bloqueador, cardiomiopatia dilatada.

\begin{abstract}
Commonly prescribed therapy for dogs presenting DCM consists of vasodilators, positive inotropic drugs (digitalics), diuretics, low-sodium diet and, when necessary, anti-arrhythmics. Carvedilol is a third generation non-selective $\beta$-blocker which blocks equally and competitively $\left(\beta_{1}, \beta_{2}\right.$ and $\left.\alpha_{1}\right)$ receptors. Produces an evident peripheral vasodilation, exerts anti-oxidative effects, removing free radicals of oxygen and preventing lipidic peroxydation of cardiac membranes, and the loss of myocytes and arrhythmias, as well as reducing mortality rate in human patients. The aim of the present study was to evaluate by physical examination, electrocardiography, radiography, and echocardiography the evolution of dogs with dilated cardiomyopathy (DCM) treated by conventional therapy associated to carvedilol. Forty-nine dogs with DCM were divided in two groups: group NT: treated with conventional therapy, and group T: treated with conventional therapy associated to carvedilol. The animals were submitted to clinical and complementary examinations during one year. The results demonstrated that carvedilol therapy presented good tolerability on the dose of $0.3 \mathrm{mg} \mathrm{kg}^{-1}$ each 12 hours, prolonged lifetime of the dogs in $30.9 \%$, did not alter systolic or diastolic pressure, reduced heart frequency after three weeks of treatment, significantly enhanced shortening and ejection fractions after six months of treatment, did not promote radiographic or $E$ septum distance alterations, decreased patients letality, as demonstrated by improvement of clinical score and functional class (heart failure according to NYHA) of the animals, obtained three weeks after the beginning of cavedilol therapy.
\end{abstract}

Key words: dogs, clinical evaluation, beta-blocker, dilated cardiomyopathy.

ICurso de Medicina Veterinária, Pontifícia Universidade Católica de Minas Gerais (PUC Minas), Rua Caminho Cinco n.20, Bairro Bortolan, 37704-403, Poços de Caldas, MG, Brasil. E-mail: mleomil@ pucpcaldas.br. Autor para corespondência.

"Universidade de São Paulo (USP), São Paulo, SP, Brasil.

IIIVeterinário Autônomo, São Paulo, SP, Brasil. 


\section{INTRODUÇÃO}

O termo cardiomiopatia (CM) denota anormalidades estruturais ou funcionais do miocárdio sendo de alta ocorrência em cães e possuindo prognóstico reservado. Essa cardiomiopatia se caracteriza por falência miocárdica biventricular (diminuição da contratilidade) e por arritmias, gerando, por sua vez, aumento dos volumes diastólico e sistólico; essa modalidade de cardiomiopatia afeta, particularmente, machos da espécie canina, de raças puras, de porte grande ou gigante, sendo a raça Doberman aquela que apresenta maior incidência, seguida pela Boxer e Cocker Spaniel (MEURS, 2005; MILLER; CALVERT, 1992).

O tratamento convencionalmente preconizado para cães acometidos pela cardiomiopatia dilatada idopática (CMD) consiste na prescrição de vasodilatadores, agentes inotrópicos positivos (digitálicos), diuréticos, dieta hipossódica e, quando necessário, antiarrítmicos.

O carvedilol, diferentemente de outros bbloqueadores, produz uma evidente vasodilatação periférica quando comparado à maioria dos agentes da mesma classe, devido ao bloqueio dos receptores a1adrenérgico (BRAUNWALD, 1997). Estes efeitos vasodilatadores podem diminuir o risco de piora da insuficiência cardíaca durante o início da terapia. Ainda, o carvedilol exerce efeitos anti-oxidantes, removendo radicais livres de oxigênio e prevenindo a peroxidação lipídica nas membranas cardíacas, prevenindo a perda de miócitos que ocorre na insuficiência cardíaca como resultado do estresse oxidativo (SAWANGKOON et al., 2000). MEURS (2005), MILLER \& CALVERT(1992) relataram que tais efeitos estão associados ao bloqueio de receptores $\beta$-adrenérgicos e também de receptores $\alpha$-adrenérgicos.

Ensaios clínicos multicêntricos, realizados em seres humanos, têm demonstrando os benefícios, como a redução da morbidade e da mortalidade, da associação de fármacos beta-bloqueadoras, como carvedilol, à terapia convencionalmente indicada para pacientes humanos com insuficiência cardíaca congestiva (ICC) (WOLLERT \& DREXLER, 2002; LECHAT, 1999; POOLE-WILSON et al., 2002).

Um estudo denominado CARIBE (CHIZZOLA et al., 2000), avaliou 30 pacientes humanos com CMD idiopática, e destes apenas dois pacientes não suportaram a dose máxima indicada e um paciente apresentou intolerância ao carvedilol. Quanto à evolução da classe funcional, dois pacientes do grupo placebo apresentaram melhora; no grupo tratado com carvedilol, 11 apresentaram melhora da classe funcional. Houve uma significante redução da frequência cardíaca (de 83 para $61 \mathrm{bpm}$ ) e aumento da fração de encurtamento e da fração de ejeção do ventrículo esquerdo no grupo carvedilol em relação ao placebo, já com dois meses de tratamento, o que se manteve até seis meses. Não ocorreram significativas alterações nas médias dos grupos durante o período de estudo quanto ao nível plasmático de catecolaminas, assim como em relação aos diâmetros do ventrículo esquerdo na sístole e na diástole, quando avaliados ecocardiograficamente.

Em recente trabalho publicado por ABBOT et al. (2005), que avaliaram os efeitos hemodinâmicos da administração oral de carvedilol em cães saudáveis, observou-se que doses de 0,08 a $0,54 \mathrm{mg} \mathrm{kg}^{-1}$, de 12 12 horas, não causaram efeitos relevantes nos animais. O carvedilol é bem absorvido pelo trato gastrintestinal e secretado pela via biliar. Além da boa tolerabilidade, o carvedilol reduz frequência cardíaca e pré-carga e aumenta o inotropismo cardíaco, em cães, quando a concentração plasmática do medicamento atinge $100 \mathrm{ng} \mathrm{ml}^{-1}$.

UECHI et al. (2002) usaram sete cães com insuficiência de valva mitral, induzida experimentalmente, e oito cães controles, todos tratados com carvedilol e submetidos à avaliação da pressão arterial, frequência cardíaca e taxa de filtração renal por método invasivo. $\mathrm{O}$ experimento demonstrou que na dose de $0,2 \mathrm{mg} \mathrm{kg}^{-1}$, 12-12horas houve redução da frequência cardíaca, porém pressão arterial e função renal permaneceram inalteradas; dobrando a dose, ou seja na dose de $0,4 \mathrm{mg} \mathrm{kg}^{-1}, 12-12$ horas, ocorreu redução de todos os parâmetros estudados, quais sejam frequência cardíaca, pressão arterial e função renal. CALVERT \& MEURS (2000), avaliando cães com CMD, observaram que o medicamento diminui os riscos de morte súbita, podendo ser indicado para quadros iniciais de cardiomiopatia do Doberman.

A despeito destes resultados encorajadores, alguns autores relatam que muitos pacientes com insuficiência cardíaca não toleram os B-bloqueadores mesmo em doses baixas. Afirmam que um percentual razoável de pacientes demostram rápida descompensação cardíaca, com piora dos sinais congestivos, porém não definem a porcentagem de pacientes com intolerância ao fármaco (BRAUNWALD, 1997).

Assim, o objetivo do presente estudo foi avaliar, clinicamente, a eficácia clínica do carvedilol no tratamento de cães com CMD idiopática, verificando, desta forma, se os dados promissores, obtidos em ensaios clínicos com seres humanos, repetem-se em espécimes caninos. 


\section{MATERIAL E MÉTODO}

Foram encaminhados ao Serviço de Cardiologia do HOVET FMVZ-USP 49 cães que apresentavam manifestações clínicas de insuficiência cardíaca, não tratados, nos quais foram realizados anamnese, exame físico, mensuração da pressão arterial, avaliação eletrocardiográfica ambulatorial, radiografias torácicas, ecocardiograma, avaliações bioquímicas, hematológicas e pesquisa de antígeno de Dirofilaria immitis.

Foram incluídos no protocolo clínico os animais com diagnóstico definitivo de CMD, sem qualquer outro quadro morbido evidente. Os animais incluídos no protocolo foram divididos, aleatoriamente, em dois grupos: grupo NT, composto por 25 cães tratados com digitálicos, diuréticos e inibidor da ECA; e o grupo $\mathrm{T}$ composto por 24 cães tratados com digitálicos, diuréticos, inibidor da ECA e betabloqueador (carvedilol - $0,3 \mathrm{mg} \mathrm{kg}^{-1}, 12-12$ horas).

Foram realizadas avaliações clínicas sucessivas, após o início do tratamento, na $3^{\mathfrak{a}}$ semana, aos 3, 6 e 12 meses, denominados, respectivamente, tempos T1, T2, T3 e T4. Por meio dos dados obtidos pela anamnese e pelo exame físico, cada animal foi incluído em uma das quatro classes funcionais da insuficiência cardíaca, segundo a New York Heart Association (NYHA).

As mensurações ecocardiográficas foram realizadas segundo a metodologia baseada na superfície corpórea do animal. Determinaram-se o volume diastólico final e o volume sistólico final do ventrículo esquerdo, o septo interventricular e a espessura do ventrículo esquerdo. Avaliaram-se o movimento da parede e a contração segmentar do ventrículo esquerdo. Foram calculadas também a função sistólica e a fração de ejeção (BOON et. al, 1983; FEIGENBAUM, 1994; LOMBARD, 1984).

Para avaliação das variáveis quantitativas contínuas (sobrevida, pressões sistólicas e diastólicas, frações de encurtamento e ejeção, VHS, frequência cardíaca e distância E-septo) utilizou-se, o Método da Máxima Verossimilhança Restrita (RELM); para as variáveis quantitativas discretas (classe funcional), utilizaram-se Modelos Lineares Generalizados.

Estas análises das estatísticas descritivas foram realizadas por meio de procedimento PROC UNIVARIATE do programa Statistical Analysis System, versão 9.1.3 (SAS, 1995).

\section{RESULTADOS E DISCUSSÃO}

Ao final do trabalho foram tratados e acompanhados, por um período máximo de 12 meses ou até o óbito do animal, 49 cães com CMD idiopática, dos quais 25 com terapia convencional (digitálico, inibidores da enzima conversora de angiotensina, diuréticos e dieta hipossódica) e os outros 24 com a terapia convencional associada ao carvedilol.

Em relação ao sexo dos animais, observouse que, do total de animais, houve um predomínio de cães machos $(73,5 \%)$ em relação às fêmeas $(26,5 \%)$, sendo, coincidentemente, 18 (dezoito) o número de machos tanto no grupo $\mathrm{T}$ (tratados com carvedilol) como NT (não tratados com carvedilol).

Apesar da inclusão dos animais nos grupos NT ou T ter sido aleatória, pode-se observar que o grupo NT foi composto por cinco cães das raças Cocker Spaniel, cinco Dobermans, quatro cães Pastores Alemães, quatro cães sem raça definida, dois da raça Boxer, dois Old English Sheepdogs e somente um componente das raças Weimaraner, Fila Brasileiro e Rottweiler, totalizando 25 cães no grupo NT. Já no grupo $\mathrm{T}$, foram estudados cinco cães da raça Dogue Alemão, quatro cães Cocker Spaniel, quatro Boxers, três Dobermans, três Old English Sheepdogs, dois Mastin Napolitanos, um cão sem raça definida e apenas um representante das raças Fila Brasileiro e São Bernardo, totalizando 24 cães no grupo $\mathrm{T}$.

A idade média dos animais pertencentes ao grupo NT foi de 8,36 anos, já do grupo T foi de 7,09 anos, não sendo observada diferença significativa entre ambas as idades médias.

No presente estudo, observou-se um predomínio de cães machos, adultos e de porte grande, como demonstrado pela literatura (MEURS (2005), MILLER \& CALVERT (1992). Analisando as raças dos animais estudados, observou-se o predomínio de cães das raças Cocker Spaniel, Doberman e Boxer, que, juntos, corresponderam a mais de $46 \%$ de todas as raças estudadas.

No presente estudo, os animais demonstraram uma boa tolerabilidade ao carvedilol quando ministrado na dose de $0,3 \mathrm{mg} \mathrm{kg}^{-1}$ de $12-12$ horas, pois não houve necessidade de excluir nenhum devido aos efeitos colaterais do fármaco. A boa tolerância ao carvedilol também é relatada por OYAMA et al. (2007) porém, em seu estudo, foi utilizada, inicialmente, a dose de $0,05 \mathrm{mg} \mathrm{kg}^{-1}$ de $12-12$ horas, com aumentos semanais na dose ministrada até atingir a dose de $0,3 \mathrm{mg} \mathrm{kg}^{-1}$ de 12-12 horas. Frente aos resultados obtidos, torna-se interessante avaliar se doses maiores ou um aumento progressivo da dose de carvedilol poderia aumentar os benefícios clínicos para cães com CMD idiopática.

O carvedilol não provocou alterações no fluxo sanguíneo dentro do coração, pois insuficiências 
valvares secundárias à dilatação cardíaca, observadas nos animais com CMD idiopática, permaneceram inalteradas, exatamente como referido por RAHKO (2005), em pacientes humanos.

Avaliou-se a influência do grupo por meio da construção das curvas de Kaplan-Meier para os dois grupos e aplicação do teste de Log-Rank para comparação estatística das duas curvas de sobrevivência (Figura 1). Os grupos foram comparados, com relação ao número de óbitos, utilizando-se o teste Exato de Fisher (ARMITAGE \& BERRY, 1987). A sobrevida e o número de animais vivos ao final do estudo são formas quantitativas de avaliar a perspectiva de vida obtida com ou sem medicamento. Do total de 25 animais do grupo NT, quatro foram avaliados até $\mathrm{T}_{3}$ e dois foram até $\mathrm{T}_{4}$; no grupo $\mathrm{T}$, dos 24 animais, cinco cães foram até $\mathrm{T}_{3}$, e seis até $\mathrm{T}_{4}$. No presente estudo, a sobrevida dos animais do grupo NT foi de $113 \pm 120$ dias, e a do grupo T de $148+135$ dias, mostrando um aumento de $30,9 \%$ na taxa média de sobrevida dos animais do grupo T em relação aos do grupo NT, sendo que, no grupo NT, dos 25 animais, dois (8\%) sobreviveram mais de um ano, período máximo de acompanhamento dos animais, enquanto que, no grupo T, dos 24 animais, seis (25\%) sobreviveram por mais de um ano. Porém, quando as médias são comparadas estatisticamente, constata-se que, face à grande variabilidade dos dados, estas não são diferentes.

Resultados semelhantes foram obtidos em pesquisas multicêntricas realizadas com seres humanos usando carvedilol e outros beta-bloqueadores, a exemplo do COPERNICUS (WOLLERT; DREXLER, 2002), em que o carvedilol reduziu em $35 \%$ a taxa de mortalidade dos cardiopatas; do CIBIS II (LECHAT, 1999), em que o bisoprolol reduziu em $20 \%$ da taxa de mortalidade; do BEST (EICHHORN et al., 2001) em que houve uma redução de $10 \%$ da taxa de mortalidade com o bucindolol; e do MERIT-HF (GHALI et al., 2002) que estudou o metoprolol e obteve uma redução de $34 \%$ na taxa de mortalidade (os trabalhos chamam de mortalidade quando deveriam chamar a taxa mensurada de taxa de letalidade. Vale ressaltar que o presente estudo conta com 49 animais avaliados por um período máximo de um ano enquanto que estudos como COPERNICUS e BEST, além de serem multicêntricos, avaliam um número bem maior de pacientes.

Quando comparamos os resultados de sobrevida do grupo NT com o grupo T, observamos que a melhora ocorreu, porém não foi significante quando comparamos com os resultados de sobrevida obtidos com outros medicamentos, como o pimobendam, por exemplo. OYAMA et al. (2007)

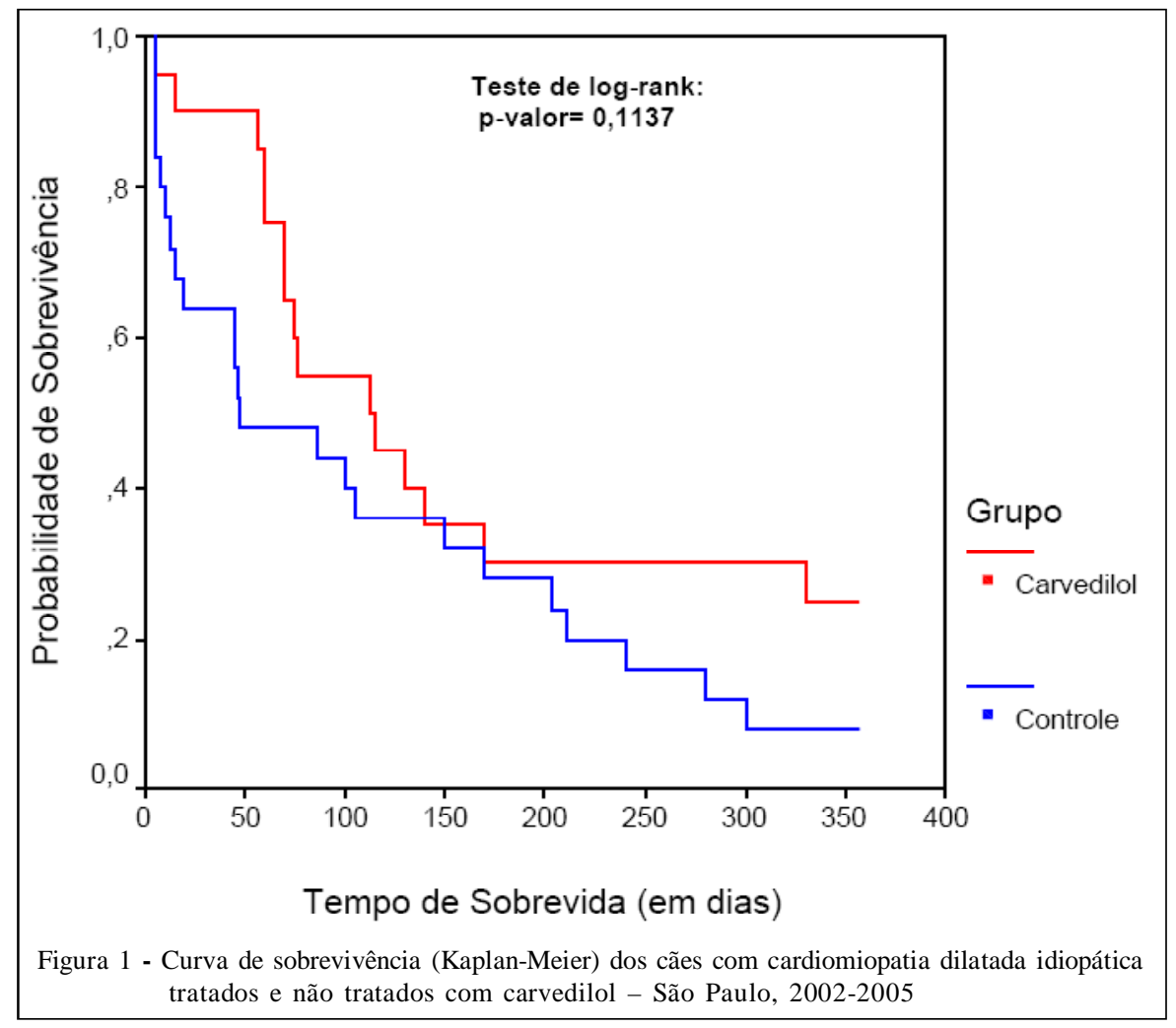

Ciência Rural, v.41, n.4, abr, 2011. 
estudaram 23 cães com CMD por quatro meses, sendo 16 com terapia convencional acrescida de carvedilol (destes 13 sobreviveram até o quarto mês) e sete com placebo substituindo o carvedilol (destes 5 animais sobreviveram até o quarto mês). Quatro cães tiveram sua terapia alterada em virtude de necessidades observadas pelos pesquisadores, demonstrando que, para alguns cães, o carvedilol contribui com melhora no sistema cardiovascular, entretanto para outros, não, e esta variação deve ser melhor estudada. Acreditamos que o carvedilol possa ser usado como mais uma opção na terapêutica dos cães com CMD compensada, protegendo o coração e os vasos sanguíneos dos efeitos deletérios da estimulação adrenérgica prolongada.

O medicamento em estudo não promoveu alterações nas pressões arteriais sistólicas (PAS) e diastólicas (PAD) quando comparado aos cães não medicados. Ao comparar os valores médios da PAS obtidos em $\mathrm{T}_{0}(124 \mathrm{mmHg})$ com $\mathrm{T}_{4}(120 \mathrm{mmHg})$ do grupo NT, e $\mathrm{T}_{0}(139 \mathrm{mmHg})$ com $\mathrm{T}_{4}(151 \mathrm{mmHg})$ do grupo $\mathrm{T}$, não há significante diferença nas médias observadas. Em relação à $\mathrm{PAD}$, os valores obtidos também não apresentaram diferenças significantes, o valor médio de PAD obtido no grupo $\mathrm{NT} \mathrm{em} \mathrm{T}_{0}$ foi de $84 \mathrm{mmHg}$, e em $\mathrm{T}_{4}$ de $80 \mathrm{mmHg}$; já no grupo $\mathrm{T}$ a média obtida em $\mathrm{T}_{0}$ foi de $92 \mathrm{mmHg}$ e em T $\mathrm{T}_{4} 94 \mathrm{mmHg}$, não havendo significante diferença nas médias observadas

Esses resultados mostraram-se interessantes pois vários autores referem-se aos efeitos vasodilatadores e inotrópico negativo do carvedilol (BRAUNWALD, 1997; LASTE, 2001), sendo que este último efeito é um dos principais motivos da apreensão demonstrada pelos pesquisadores na utilização de bloqueadores $\beta$-adrenérgicos em casos de CMD idiopática. Frente aos resultados obtidos, pode-se afirmar que, mesmo com os efeitos vasodilatador e inotrópico negativo induzidos pelos $\beta$-bloqueadores, o carvedilol foi bem tolerado pelo sistema cardiovascular, conseguindo manter uma relação adequada entre o débito cardíaco e a resistência vascular periférica, suficiente para manter dentro dos padrões de normalidade as pressões arteriais sistólica e diastólica dos cães medicados.

Os resultados relativos à frequência cardíaca (FC) demonstraram que não houve diferença significante na média do referido parâmetro quando comparamos o grupo T e NT, ou seja, cães tratados e não tratados com carvedilol iniciaram o protocolo com uma FC estatisticamente igual e mantiveram-na por todo o período de avaliação. Observou-se, entretanto, uma redução das médias de $\mathrm{FC}$ nos dois grupos com o decorrer do tempo; porém, quando da comparação das médias, dentro do próprio grupo, no grupo NT, houve uma redução gradual entre as médias de $\mathrm{T}_{0}, \mathrm{~T}_{1}, \mathrm{~T}_{2}, \mathrm{~T}_{3} \mathrm{e}$ $\mathrm{T}_{4}$, caracterizando, portanto, uma queda gradativa na FC. Já no grupo $T$, houve diferença significativa entre as médias de $\mathrm{T}_{0} \mathrm{e} \mathrm{T}_{1}, \mathrm{~T}_{0} \mathrm{e} \mathrm{T}_{2}, \mathrm{~T}_{0}$ e $\mathrm{T}_{3} \mathrm{eT}_{0} \mathrm{e} \mathrm{T}_{4}$, indicando uma queda inicial da $\mathrm{FC}$ (entre $\mathrm{T}_{0}$ e $\mathrm{T}_{1}$ ) e a posterior manutenção desta queda inicial, pois não houve diferença entre as médias de $T_{1}, T_{2}, T_{3}$, e $T_{4}$.

Não se pode afirmar que essa redução súbita da frequência cardíaca observada no grupo $\mathrm{T}$ tenha efeitos deletérios para o sistema cardiovascular pois, mesmo com a diminuição do referido parâmetro eletrocardiográfico, não houve alterações na pressão arterial e tampouco piora clínica dos animais. Vale ressaltar que, para animais com um grau mais avançado de insuficiência miocárdica, essa redução da frequência cardíaca pode levar a reduções importantes do débito cardíaco e, consequentemente, piora do quadro clínico do animal.

RAHKO (2005) estudou pacientes humanos com insuficiência cardíaca moderada a severa e observou que o carvedilol aumenta as frações de ejeção e de encurtamento. CHIZZOLA et al. (2000), avaliando 30 pacientes humanos, obteve um aumento da média da fração de encurtamento de $13,3 \%$ para $15,2 \%$. Embora no presente trabalho a espécie estudada seja diversa daquela avaliada por CHIZZOLA et al. (2000) e RAHKO (2005), pode-se perceber que os resultados obtidos no presente trabalho são semelhantes àqueles observados pelos referidos pesquisadores, em seres humanos, pois as frações de encurtamento e ejeção apresentaram melhora significante $(\mathrm{P}<0,05)$, isto é, enquanto animais do grupo NT tiveram um aumento da fração de encurtamento de $16,02 \%$, em $\mathrm{T}_{0}$, para $18,34 \%$ em $\mathrm{T}_{4}$, os animais do grupo $\mathrm{T}$ apresentaram média de $16,13 \%$ em $\mathrm{T}_{0}$ e, após um ano de tratamento $\left(\mathrm{T}_{4}\right)$, passaram para $25,10 \%$ de média. Em relação à fração de ejeção, também se observou um significante aumento em decorrência da adição do carvedilol ao tratamento convencional, pois o grupo NT, com média de $31,92 \%$ em $\mathrm{T}_{0}$, passou para $36,14 \% \mathrm{em} \mathrm{T}_{4}$, enquanto que no grupo tratado houve uma melhora significativa de $32,63 \%$, em $\mathrm{T}_{0}$, para $44,95 \%$, em $\mathrm{T}_{4}$. Ao analisar os dados tanto da fração de encurtamento como de ejeção, nota-se que, principalmente após o $\mathrm{T}_{3}$, ou seja, decorridos seis meses do início do tratamento com carvedilol, é que a melhora das frações se torna mais evidente. OYAMA et al. (2007) não observaram alterações nos principais parâmetros ecocardiográficos em cães medicados com carvedilol nos primeiros quatro meses de tratamento, o que corrobora os dados obtidos no presente estudo. Em seres humanos o aumento da fração de encurtamento do ventrículo esquerdo ocorre, 
segundo o estudo denominado CARIBE (CHIZZOLA et al., 2000), após dois meses de tratamento com carvedilol, porém, no estudo, os autores tratavam os pacientes com a dose máxima indicada ou suportada pelo paciente, enquanto que, no presente estudo, a dose foi intermediária, não havendo aumentos no decorrer do tratamento.

O ponto máximo do movimento de abertura do folheto septal ou anterior da valva mitral (ponto E) ao septo interventricular, a chamada distância E-septo, não sofreu alterações significativas no decorrer do estudo no grupo dos cães tratados assim como no grupo dos não tratados com carvedilol.

Quanto ao VHS (vertebral heart size) não houve modificação nos valores apurados com o decorrer do tempo nos animais com CMD idiopática, tanto no grupo dos cães tratados $\left(\mathrm{T}_{0}=12,35 ; \mathrm{T}_{1}=12,40\right.$; $\mathrm{T}_{2}=12,61 ; \mathrm{T}_{3}=12,92$ e $\left._{4}=12,40\right)$ como nos nãotratados com carvedilol $\left(\mathrm{T}_{0}=12,43 ; \mathrm{T}_{1}=12,45 ; \mathrm{T}_{2}=12,30 ; \mathrm{T}_{3}=12,50\right.$ $\mathrm{e}_{4}=12,40$ ).

O VHS e a distância E-septo apresentam uma relação direta e proporcional com a pressão intracardíaca e, consequentemente, com o diâmetro cardíaco (BOON, 1998). Pode-se supor que, apesar da melhora clínica observada, não houve uma redução da silhueta e nem da cavidade cardíaca nos animais medicados com carvedilol, provavelmente por não ter ocorrido redução significante na pré-carga (pressão intracavitária) a ponto de reduzir o diâmetro cardíaco. Um ponto interessante a ser abordado é que autores relatam efeitos inotrópico e cronotrópico negativos em animais e pessoas medicadas com carvedilol (BRAUNWALD, 1997; CALVERT \& MEURS, 2000), porém, se tal efeito ocorresse nos animais do estudo, o VHS e a distância E-septo teriam aumentado, demonstrando um aumento proporcional da pré-carga e do quadro congestivo. Evidentemente estudos hemodinâmicos devem se realizados, permitindo aferir, de forma fidedigna, o comportamento da pressão intracavitária cardíaca durante a evolução da CMD idiopática em animais tratados com carvedilol ou não. Porém pode-se aventar a hipótese de que o carvedilol, no conjunto de suas ações, não altera a hemodinâmica cardíaca, mantendo-a igual à de animais não medicados com carvedilol.

Quanto à evolução da classe funcional, observou-se que $75 \%$ dos animais do grupo T e $40 \%$ do grupo NT apresentaram melhora de classe funcional, observa-se um maior número de animais com melhora na classe funcional no grupo T; por outro lado $25 \%$ do grupo T e 56\% do grupo NT não sofreram alterações de classe funcional, permitindo-nos afirmar que a ausência do carvedilol impediu que um número elevado de animais melhorasse de classe funcional. Fica claro, baseado na classe funcional, que o carvedilol associado à terapia convencional melhorou, clinicamente, a maior parte dos cães acometidos pela CMD idiopática, principalmente em cães que não apresentam processos congestivos. $O$ trabalho multicêntrico CARIBE (CHIZZOLA et al., 2000) relata resultados semelhantes, pois, no grupo placebo, cinco pacientes permaneceram inalterados e dois apresentaram melhora em relação à classe funcional, ao passo que, medicados com carvedilol, quatro pacientes permaneceram inalterados e 11 melhoraram de classe funcional. BOCCHI (1998), em ensaios com seres humanos, também observou redução da classe funcional em pacientes medicados com carvedilol.

\section{CONCLUSÃO}

Fundamentando-se nos resultados obtidos no presente estudo, para as condições em que foi desenvolvido, concluiu-se que: o $\beta$-bloqueador carvedilol, na dose de $0,3 \mathrm{mg} \mathrm{kg}^{-1}, 12-12$ horas, foi seguro e bem tolerado pelos animais que recebem a medicação. Além disso, a adição do carvedilol à terapia, convencionalmente indicada para cães com CMD idiopática, resultou em aumento de $30,9 \%$ da sobrevida; não causou alterações significantes nas pressões arteriais sistólica e diastólica, VHS e na distância Esepto; reduziu de forma marcante a frequência cardíaca, nos primeiros 21 dias de tratamento; provocou aumento das frações de ejeção e de encurtamento, a partir de 180 dias do início da terapia; além de causar reduções da classe funcional (segundo NYHA).

\section{REFERÊNCIAS}

ABBOT, J.A. et al. Hemodynamic effects of orally administered carvedilol in healthy conscious dogs. American Journal of Veterinary Research, v.66, n.4, p.637-41, 2005. Disponível em: <http://avmajournals.avma.org/doi/ abs/10.2460/ajvr.2005.66.637>. Acesso em: 19 jan. 2011. doi: 10.2460/ajvr.2005.66.637.

ARMITAGE, P.; BERRY, G. Statistical methods in medical research. 3.ed. Oxford: Blackwell Science, 1994. 832p.

BRAUNWALD, E. Tratado de medicina cardiovascular. 5.ed. São Paulo: Roca, 1997. 2248p.

BOCCHI, F.A. et al. Effects of carvedilo (beta1, beta 2, alpha 1 bloker) on refractory congestive heart failure. Arquivos Brasileiros de Cardiologia, v.71, n.2, p.169-73, 1998. Disponível em: <http://www.scielo.br/ scielo.php?pid=S0066782X1998000800014\&script $=$ sci_arttext $>$. Acesso em: 19 jan. 2011. doi: 10.1590/S0066-782X1998000800014.

BOON J.A. Acquired heart disease. In BOON, J.A. Manual of veterinary echocardiography. Baltimore: Williams \& Wilkins, 1998. p.261-382. 
BOON, J.A. et al. Echocardiographic indices in the normal dog. Veterinary Radiology, v.24, n.2, p.214, 1983. Disponível em: <http://onlinelibrary.wiley.com/doi/10.1111/j.17408261.1983tb00718.x/abstract>. Acesso em: 19 jan. 2011. doi: 10.1111/j.1740-8261.1983.tb00718.x.

CARVERT,C. A.; MEURS, K. CVT: update: Doberman Pincher occult cardiomyopathy. In: BONAGURA, J.D. Kirk's current veterinary therapy - Small animal pratice. Philadelphia: Saunders, 2000. p.756-760.

CHIZZOLA, P.R. et al. Efeitos do carvedilol na insuficiência cardíaca por cardiomiopatia dilatada. Resultado de estudo duplocego, randômico, com grupo placebo-controle (ESTUDO CARIBE). Arquivo Brasileiro de Cardiologia, v.74, n.3, p.233-37, 2000. Disponível em: <http://bases.bireme.br/cgibin/wxislind.exe/iah/online/? Isis Script=iah/ iah. $x i s \& s r c=$ google \&base $=$ LILACS\&lang $=$ p\&nextAction $=1 n k \& e$ $x$ prSearch $=265165 \&$ indexSearch=ID $>$. Acesso em: 19 jan. 2011. doi: indentificador único 265165 .

EICHHORN, E.J. et al. A trial of beta-blocker bucindolol in patients with advanced chronic heart failure. New England Journal of Medicine, v.344, n.22, p.1659-67, 2001. Disponível em: 〈http://www.ncbi.nlm.nih.gov/pubmed/11386264〉. Acesso em: 19 jan. 2011. doi: PMID- 11386264.

FEIGENBAUM, A. Echocardiography. 5.ed. Philadelphia: Lea and Febiger, 1994. 695p.

GHALI, J.K. et al. MERIT - HF Study Group Metoprolol CR/ $\mathrm{XL}$ in female patients with heart failure: analysis of the experience in metoprolol extended-release randomized intervention trial in heart failure (MERIT - HF). Circulation, v.105, n.13, p.1585-1591, 2002. Disponível em: <http:// www.ncbi.nlm.nih.gov/pubmed/11927527>. Acesso em: 19 jan. 2011. doi: PMID - 1585-91.

LASTE, N.J. Cardiovascular pharmacotherapy. Veterinary Clinics of North America: Small Animal Practice, v.31, n.6, p.1231-1254, 2001. Disponível em: <http://www.ncbi.nlm.nih.gov/ pubmed/11727335>. Acesso em: 19 jan. 2011. doi: PMID 1231-52.

LECHAT, P. A cardiac insufficiency bisoprolol study II (CIBIS II): a randomized trial. Lancet, v.353, n.9146, p.9-13, 1999. Disponível em: 〈http://www.ncbi.nlm.nih.gov/pubmed/10023943〉. Acesso em: 22 jan. 2011. doi: PMID - 10023943.

LOMBARD, C.W. Normal values of the canine M mode echocardiogram. American Journal of Veterinary Research, v.45, n.10, p.2015, 1984. Disponível em: <http:/ / w w w.s cielo.br/s c i e lo.ph p ? pid=S $0103-$ 84782000000500013\&script=sci_arttext $>$. Acesso em: 22 jan. 2011. doi: PMID- 6497098.

MEURS, K.M. Primary myocardial disease intha dog. In: ETTINGER, S.J.; FELDMAN, E.C. (Ed.) Textbook of veterinary internal medicine. 6.ed. St. Louis: Elsevier Saunders, 2005. V.2, p.1077-1081.

MILLER, M.S.; CALVERT, C.A. Special methods for diagnosing arrhythmias. In: TILLEY, L.P. Essentials of canine and feline electrocardiography. 3.ed. Philadelphia: Lea \& Febiger, 1992. p.289-319.

OYAMA, M.A. et al. Carvedilol in dogs with dilated cardiomyopathy. Journal Veterinary Internal Medicine, v.21, p.1272-1279, 2007. Disponível em: <http:// onlinelibrary.wiley.com/doi/10.1111/jvim.2007.21.issue-6/ issuetoc>. Acesso em: 22 jan. 2011. doi: 10.1111/j.19391676.2007.tb01949.x.

POOLE-WILSON, P.A. et al. Rationale and design of the carvedilol or metoprolol European trial in patients with chronic heart failure: COMET. European Journal Heart Failure, v.4, n.3, p.321-329, 2002. Disponível em: <http:// www.ncbi.nlm.nih.gov/pubmed/12034158>. Acesso em: 22 jan. 2011. doi: PMID - 12034158.

RAHKO, P.S. An echocardiographic analysis of the long-term effects of carvedilol on left ventricular remodeling systolic performance, and ventricular filling patterns in dilated cardiomyopathy. Echocardiography, v.22, n.7, p.547-554, 2005. Disponível em: <http://www.ncbi.nlm.nih.gov/pubmed/ 16060890>. Acesso em: 22 jan. 2011. doi: PMID - 16060890.

SAS. USER'S GUIDE: Basic and Statistic. Cary, 1995. 1686p.

SAWANGKOON, S. et al. Accute cardiovascular effects and pharmacokinectis of carvedilol in healthy dogs. American Journal of Veterinary Research, v.61, n.1, p.57-60, 2000. Disponível em: <http://avmajournals.avma.org/doi/abs/10.2460/ ajvr.2000.61.57>. Acesso em: 22 jan. 2011. doi: 10.2460/ ajvr.2000.61.57.

UECHI, M. et al. Cardiovascular and renal effects of carvedilol in dogs with heart failure. Journal of Veterinary Medicine Science, v.64, n.6, p.469-475, 2002. Disponível em: <http:/ /www. ncbi.nlm.nih.gov/pubmed/12130829>. Acesso em: 22 jan. 2011. doi: PMID- 12130829.

WOLLERT, K.C.; DREXLER, H. Carvedilol prospective randomized cumulative survival trial. Circulation, v.106, n.17, p.2164-2166, 2002. Disponível em:<http:// circ.ahajournals.org/cgi/ content/full/106/17/2164>. Acesso em: 22 jan. 2011. doi: 10.1161/01.CIR.0000038702.35084.D6. 